\title{
Feature Extraction Method GLCM and LVQ in Digital Image-Based Face Recognition
}

\author{
T. Sukma Achriadi Sukiman \\ University of north Sumatra \\ Medan, Indonesia \\ adimarlago@Gmail.com
}

\author{
Prof. Dr. Saib Suwilo \\ University of north Sumatra \\ Medan, Indonesia
}

\author{
Prof. Dr. Muhammad Zarlis \\ University of north Sumatra \\ Medan, Indonesia
}

\begin{abstract}
The face is one of the media to identify someone, a human face has a very high level of variability. Many methods have been introduced by researchers and scientists in recognizing one's face, one of the methods introduced is the Feature Extraction of Gray Level Co-Occurrence Matrix (GLCM) and Learning Vector Quantization (LVQ). GLCM feature extraction is used for data extraction/learning process whereas a data analysis process (face recognition, cropping and storing data) the LVQ method is used for the data training process where the data that has been processed in GLCM feature extraction which still has large dimensions are processed to be smaller dimensions. So this test uses data of 190 photos and gets a match of $90 \%$, the authors conclude that the GLCM feature extraction and LVQ method can very well recognize faces contained in the database.
\end{abstract}

Keywords - GLCM, $L V Q$, Citra Digital

\section{INTRODUCTION}

Face is one of biometry that can define a person, the human face is a dynamic object that has a high level of variability. Many studies of facial recognition, one of which is a facial recognition system that utilizes a computer is not a simple system (Choong Hwan Lee, 1996). Furthermore, human face recognition by using a collection of still images or videos with a set of videos (Zhou, 2004). With the use of video cameras and computers it is good enough to process video in real-time (Gorodnichy, 2004) face recognition uses a camera to capture a person's face and after that compared to a face that has previously been stored in a database in real-time (Bayu, 2009). The use of the Gray Level Co-insurance Matrix (Gray Level Co-Occurrence Matrix / GLCM feature extraction) is mostly done in taking remote sensing imagery with prototypes (Maheshwary, 2009). The development of biometric technology such as face, voice, iris, and fingerprints is very much developed both as a security system and in the present system. Biometric technology has been developed and applied in various applications, but in fact, the recognition process sometimes still fails. Among the failures are caused by lighting factors, the distance of the object to the tool, the angle of the object to the tool, the expression and face position. In this study, an application was built to measure the accuracy of facial recognition with the Gray Level Co-matrix Extraction Feature of the Gray Level Co-Occurrence Matrix (GLCM) and Learning Vector Quantization (LVQ) Artificial Neural Networks in different light intensities, distances, and angles.

\section{LITERATURE REVIEW}

1. Face

The human face plays a central role in social interaction, therefore it is not surprising that automatic face information processing is an important subfield and is very active in pattern recognition research (j. Lyons, 1999). The face displays a variety of complicated information about identity, age, gender, race, and emotional state and attention. One of them is facial expression. Expression is an expression of feeling, which can be shown through a movement, be it the movement of the hands, feet, voice or face. (Muhathir, 2018)

\section{Digital Image}

Digital image is a function of two variables, $f(x, y)$, where $\mathrm{x}$ and $\mathrm{y}$ are the spatial coordinates and the value $f(x, y)$ which is the intensity of the image in these coordinates. The basic technology in creating and displaying colors in digital images based on research is a color that is a combination based on three basic colors, namely red, green, and blue (Red, Green, Blue - RGB). 


\section{Gray Level Co-occurrence Matrix}

Gray Level Co-occurrence Matrix is a matrix whose elements are the number of pixel pairs that have a certain brightness level, where the pixel pairs are separated by distance (d) and angle $(\Theta)$ (). Gray Level Co-occurrence Matrix is a method used to obtain a feature of an image that later results from the search for the characteristics of an image that can be used as an input to classify the image into certain groups or classes that have been previously agreed upon. Steps to get feature extraction by extracting Gray Level Co-occurrence features are as follows:

a. The first step is to create a matrix work area because the image has four gray levels, therefore the number of neighboring pixel values and reference pixel values in the matrix work area are four. The following is the matrix work area that can be seen in Figure 1.

\begin{tabular}{|c|c|c|c|c|}
\hline Piksel tetangga & & 1 & 2 & 3 \\
\hline 0 & 0,0 & 0,1 & 0,2 & 0,3 \\
\hline 1 & 1,0 & 1,1 & 1,2 & 1,3 \\
\hline 2 & 2,0 & 2,1 & 2,2 & 2,3 \\
\hline 3 & 3,0 & 3,1 & 3,2 & 3,3 \\
\hline
\end{tabular}

Picture 1. Matrix work area

b. The second step is to determine the angle $(\Theta)$, and distance (d) to determine the spatial relationship between the reference pixel and neighboring pixels, determined by the angle $(\Theta)=0$ and distance $(d)=1$. The orientation angle determines the direction of the relationship of the neighboring pixels to the pixel reference pixel. The orientation of the angle $(\Theta)=0$ means the horizontal direction, as explained in Figure 2:

\begin{tabular}{|c|c|c|c|c|c|c|c|c|}
\hline 0 & 0 & 1 & 1 & 0 & 0 & 1 & 2 & 3 \\
\hline 0 & 0 & 1 & 1 \\
\hline 0 & 2 & 2 & 2 & 2 & 1 & 0 \\
\hline 2 & 2 & 3 & 3 & 0 & 2 & 0 & 0 \\
\hline & 3 & 0 & 0 & 3 & 1 \\
\hline & 0 & 0 & 0 & $>1$ \\
\hline
\end{tabular}

Figure 2 Spatial relationship between pixels

c. The next step is to add the cohesion matrix to the transpose matrix to get a symmetrical matrix, as shown in Figure 3:

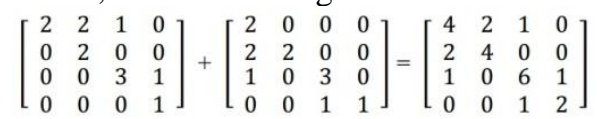

Figure 3. Symmetric matrix d. After getting a symmetrical matrix value, the next step is to normalize the matrix into probabilities. The trick is that each cell is divided by the sum of all spatial elements, as shown in the following image:

$$
\left[\begin{array}{cccc}
\frac{4}{24} & \frac{2}{24} & \frac{1}{24} & \frac{0}{24} \\
\frac{2}{24} & \frac{4}{24} & \frac{0}{24} & \frac{0}{24} \\
\frac{1}{24} & \frac{0}{24} & \frac{6}{24} & \frac{1}{24} \\
\frac{0}{24} & \frac{0}{24} & \frac{1}{24} & \frac{2}{24}
\end{array}\right]=
$$

\begin{tabular}{|c|c|c|c|}
\hline 0,1667 & 0,0833 & 0,0416 & 0 \\
\hline 0,0833 & 0,1667 & 0 & 0 \\
\hline 0,0416 & 0 & 0,25 & 0,0416 \\
\hline 0 & 0 & 0,0416 & 0,0833 \\
\hline
\end{tabular}

Figure 4. Normalization of the matrix with probability

e. After getting the value from the image cohesion matrix used, it can be calculated statistical features that represent the image that has been used or observed. For the features that have been extracted from the image coherence matrix observed using 4 features, namely, Correlation, Energy, Homogeneity, and Contrast.

Correlation is a linear gray tone dependence on an observed image. Then $P(i, j)$ is the probability distribution along with pixel pairs of gray levels $i$ and $\mathrm{j}$ for the cohesion matrix. Correlation is the average pixel value and is a standard deviation (Dewi, 2014). For the search equation formula from Correlation is as follows:

Korelasi $=\frac{\sum_{i=1} \sum_{j-1}(i=\mu i)(j=\mu j)(\operatorname{GLCM}(i, j))}{\sigma i \sigma j}$

Energy is a measurement of the distribution of pixel intensity over the gray level range of an observed image. Where $P(i, j)$ is the value of row too $\mathrm{i}$ and column to $\mathrm{j}$ in the cohesion matrix. For the search equation formula from Energy are as follows: Energy $=\sum_{i=1} \sum_{j=1} \operatorname{GLCM}(i, j)^{2}$

Homogeneity is the similarity or homogeneity of variations of the cohesion matrix of the observed image. For the search equation formula from homogeneity is as follows:

Homogenitas $=\sum_{i=1} \sum_{j=1} \operatorname{GLCM}(i, j)^{2}$

Contrast is to show the spread (moment of inertia) of the elements of the image matrix. For the search equation the formula of Contrast is as follows: 
Kontras $=\sum_{i=1} \sum_{j=1}|i-j|^{2} * \operatorname{GLCM}(i, j) \ldots \ldots \ldots . .(4)$

\section{Metode Learning Vektor Quantization}

Learning vector quantization (LVQ is one of the methods to conduct supervised competitive learning. This Competitive Layer will learn automatically to classify the given input vector. If two vectors have close distances will be grouped into one same class (Kusumadewi, 2004). The LVQ network has the first competitive layer and the second linear layer, the competitive layer learns to classify the vector to be entered in. The linear layer changes the competitive layer class to the target classification to be defined by the user. classes are studied by competitive layers as subclasses and classes from linear layers into target classes (Demuth, 2002).

\section{Proposed Method}

\section{Datasheet}

The data used in this study are face data from someone where required 5 types of photos in jpg format, in which all types of photos are photos of faces facing front (frontal) $0 \mathrm{o}$ facing to the left $25 \mathrm{o}$ and $75 \mathrm{o}$, then facing to the right $-25 \mathrm{o}$ and $-75 \mathrm{o}$, not partially blocked by other objects and also visible both eyeballs.

2. Research step

The steps taken in this study are as follows:

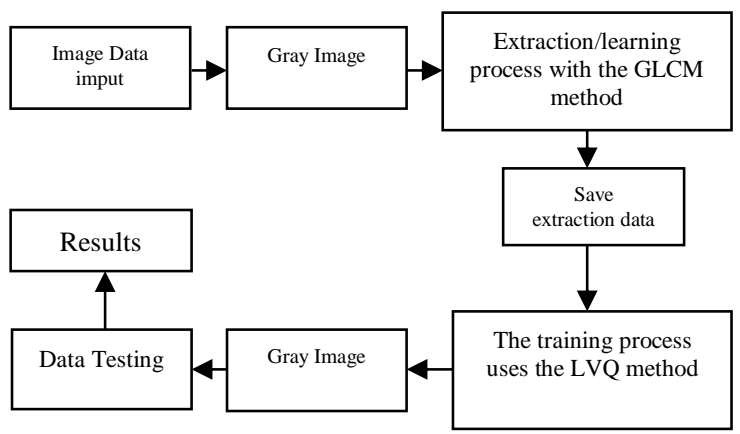

Figure 5. General research steps

in the picture above shows the flow of the process and the steps of this research, the first process is the process of extraction or introduction using the GLCM method, after the image data is processed by the GLCM method, the data will be stored in a database and continued with the training process using the LVQ method, where all the stored image data will be collected and a distinction will be made between the whole saved image data. And for the test in this study, researchers used the LVQ method to try to find accuracy in facial recognition using the two methods mentioned earlier.
The steps carried out in the research as a whole can be illustrated in the picture :

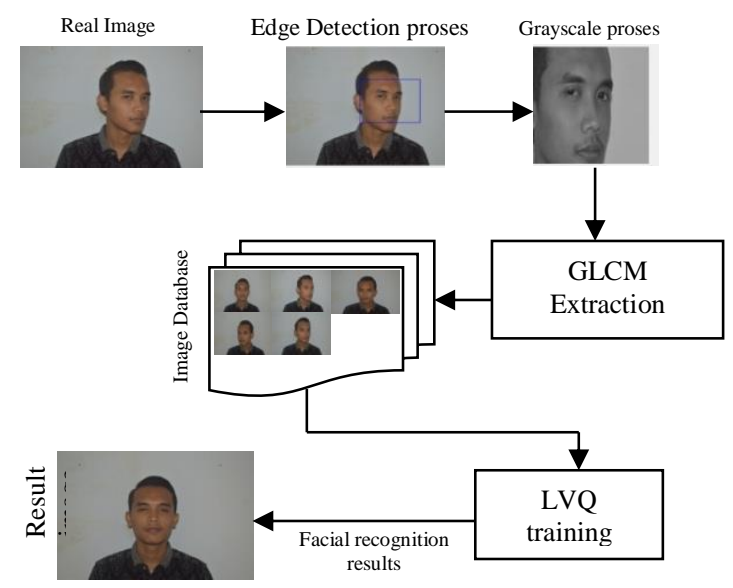

Figure 6 Research steps

\section{RESULT AND DISCUSSION}

1. Facial training/extraction samples

The sample used in this study is 190 images of 38 people facing forward (frontal) $0 \mathrm{o}$ facing left $25 \mathrm{o}$ and $75 \mathrm{o}$, then facing right $-25 \mathrm{o}$ and $-75 \mathrm{o}$, not partially blocked by other objects and also seen both eyeballs, the picture shows some sample data that are tested are as follows:

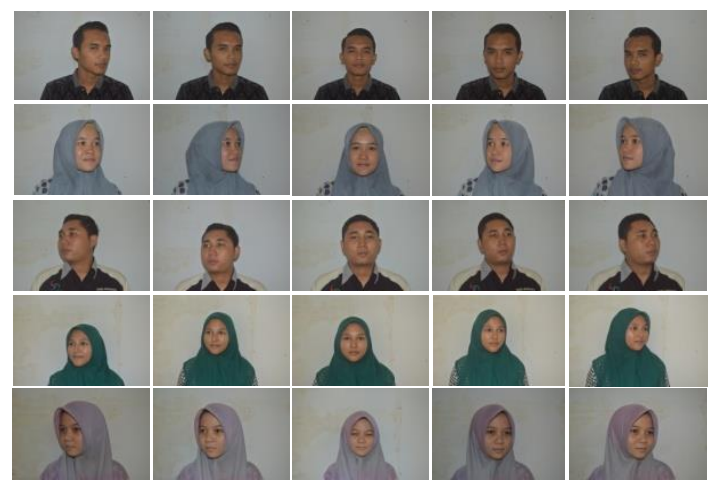

2. Test results of GLCM and LVQ extraction accuracy

The results of tests conducted to study the accuracy in face recognition using the GLCM and LVQ methods are as follows:

\begin{tabular}{|c|c|c|}
\hline Samples & Recognized & Unrecognized \\
\hline 20f & 4 & 1 \\
\hline
\end{tabular}




\begin{tabular}{|c|c|c|}
\hline & 5 & - \\
\hline & 2 & 3 \\
\hline & 4 & 1 \\
\hline & 5 & - \\
\hline & 5 & - \\
\hline & 4 & 1 \\
\hline & 5 & - \\
\hline & 5 & - \\
\hline & 5 & - \\
\hline & 5 & - \\
\hline : & .•• & $\cdots$ \\
\hline & 5 & - \\
\hline & 5 & - \\
\hline
\end{tabular}

\section{Discussion}

Based on the results of testing in this study, where the data tested were 190 images or 35 human faces, there were 171 results identified while the unrecognized data were 19 , with the percentage of data recognition $90 \%$.

\section{Conclusionand Suggestion}

The results of the research that have been done show that the extraction and LVQ method can properly recognize the faces in the test, while the thing that makes the image unrecognizable due to blurry images, and is too dark in the lighting and also causes the image to be unrecognized due to the existing object in the edge image is not drawn for example the edge of the eye from the object object.

\section{REFERENCES}

Bayu, S. H. (2009). Penerapan Face Recognition Dengan Metode Eigenface dalam Intelligent Home Security.

Choong Hwan Lee, J. S. (1996). Automatic Human Face Location in a Complex Background Using Motion and Color Information. Pattern Recognition, 27.

Demuth, h. a. (2002). Neural Network Toolbox User's Guide. The MathWork.

Dewi, R. H. (2014). Identifikasi Penyakit Pada Daun Tebu Dengan Gray Level Co-occurrence Matrix dan Color Moments. Teknologi Informasi dan Ilmu Komputer (JTIIK), 2, 70-77.

Gorodnichy, D. (2004). Introduction to the First IEEE Workshop on Face Processing in Video. Conference Publications, 27.

j. Lyons, M. B. (1999). Automatic Classification of Single Facial Images. Pattern Analysis and Machine Intelligence, 21, 1357-1362.

Kusumadewi, S. (2004). Membangun Jaringan Syaraf Tiruan Menggunakan Matlab dan Excellink.

Maheshwary, P. d. (2009). Prototype System for Retrieval of Remote Sensing Images based on Color Moment and Gray Level CoOccurrence Matrix. IJCSI International Journal of Computer Science Issues, 3.

Muhathir, M. (2018). KLASIFIKASI EKSPRESI WAJAH MENGGUNAKAN BAG OF VISUAL. JITE (JOURNAL OF INFORMATICS AND TELECOMMUNICATION ENGINEERING), 1(2), 73-82.

Zhou, S. K. (2004). Probabilistic recognition of human faces from video. Computer Vision and Image Understanding. 91. 214-245. 